Proceedings of the 13th International Symposium UFPS, Vilnius, Lithuania 2007

\title{
Fast Electrical Switching of Thin Manganite Films
}

\author{
S. BAleviČIUS ${ }^{a, b}$, O. Kiprijanovič ${ }^{a}$, V. StankeviČ ${ }^{a, b}$,

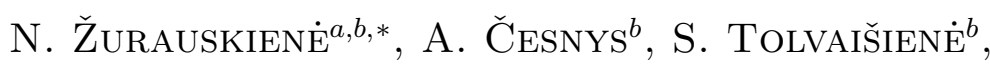 \\ A. Abrutis ${ }^{c}$ And V. Plaušinaitiene $\dot{E}^{a, c}$
}

${ }^{a}$ Semiconductor Physics Institute, A. Goštauto 11, Vilnius, Lithuania

${ }^{b}$ Vilnius Gediminas Technical University

Sauletekio 11, Vilnius, Lithuania

${ }^{c}$ Vilnius University, Dep. of General and Inorganic Chemistry

Naugarduko 24, Vilnius, Lithuania

The effects of strong pulsed electric field on the electrical properties of thin epitaxial $\mathrm{La}_{0.7} \mathrm{Sr}_{0.3} \mathrm{MnO}_{3}$ films were investigated. The fast electrical switching from high resistance off-state to low resistance on-state was obtained at current densities higher than $10^{6} \mathrm{~A} / \mathrm{cm}^{2}$. This current was able to induce an irreversible damage of the sample in the regions at the edges of the electrodes of the film. It was demonstrated that thermal effects are responsible for appearance of delay time and asymmetrical shape of current channel in on-state, however, the fast switching from off- to on-state is a result of electronic effects appearing when critical power is reached in the film.

PACS numbers: 75.47.Lx, 75.47.Gk, 73.50.Fq

\section{Introduction}

Recently, an interest to high current effects in manganites had increased [1]. It was obtained that high current induces an electrical instability (switching) in thin polycrystalline manganite film, the process during which the resistance of the film suddenly decreases several times. As a result, the irreversible damaging of the device occurs mainly at the electrode regions. This phenomenon is very important for designing protectors against short electromagnetic pulse (SEMP) [2], because it increases the attenuation in protecting regime, but at the same time it also limits the highest possible power of device operation. In order to reveal the nature and behavior of the switching we investigated high current regime in epitaxial films having the same chemical composition as polycrystalline films used for protectors against SEMP.

\section{Film preparation technology}

The investigated samples were made from $\mathrm{La}_{0.83} \mathrm{Sr}_{0.17} \mathrm{MnO}_{3}$ films which had been prepared using metal organic chemical vapour deposition (MOCVD) from a

*corresponding author; e-mail: zurausk@pfi.lt 
liquid source solution having the composition $\mathrm{La}_{0.78} \mathrm{Sr}_{0.22} \mathrm{Mn}_{0.733}$ [3]. The epitaxial films were grown on $\mathrm{NdGaO}_{3}$ substrate at $825^{\circ} \mathrm{C}$. Thickness of the films was $400 \mathrm{~nm}$. Samples had a co-planar microbridge shape with the $0.5 \times 0.5 \mathrm{~mm}$ square Ag electrodes spaced each to the other at $d=50 \mu \mathrm{m}$ distance. The width $(w)$ of the interelectrode material varied from 0.1 to $0.5 \mathrm{~mm}$. Phase transition temperature $T_{\mathrm{m}}$ obtained from low-field dc measurements was $330 \mathrm{~K}$. For electric field induced resistance change measurements the samples were connected in series to a $50 \Omega$ impedance $12 \mathrm{GHz}$ frequency transmission line and affected by $10-18 \mathrm{~ns}$ duration and 0.5 ns rise time, rectangular shape single electrical pulses with amplitudes up to $1 \mathrm{kV}$ [4]. The dynamics of the switching was recorded by real time $5 \mathrm{GHz}$ oscilloscope. The surface morphology of irreversibly damaged films was studied by optical and scanning electron microscopes (SEM).

\section{Results and discussion}

Typical current-voltage characteristics of epitaxial film measured at different time instants $\left(\tau_{\mathrm{i}}\right)$ at temperature $T=295 \mathrm{~K}$ are shown in Fig. 1a. They are nonlinear in positive differential resistance region up to the threshold voltage $\left(V_{\mathrm{th}}\right)$ at which switching from high resistance (off-state) to low resistance (on-state) occurred. The shape of $I-V$ curves at $V<V_{\text {th }}$ corresponding to different $\tau_{\mathrm{i}}$ is a result of the Joule heating and resistivity vs. temperature dependence of epitaxial film exhibiting phase transition from ferromagnetic to paramagnetic state [4]. At $\tau_{\mathrm{i}}=2 \mathrm{~ns}$ the heating is relatively small and $I-V$ curve up to $175 \mathrm{~V}$ demonstrates the increase in the film resistance which means that the temperature of the film is less than resistivity maximum temperature $T_{\mathrm{m}}$. For higher voltages the temperature growth of the film is accompanied with resistance decrease as in this case $T>T_{\mathrm{m}}$. At longer times $\tau_{\mathrm{i}}=5 \mathrm{~ns}$ the heating even at $50 \mathrm{~V}$ is sufficient to transform the film from ferromagnetic to paramagnetic state. For this reason at $V>50 \mathrm{~V}$ the temperature of the film after $5 \mathrm{~ns}$ is always higher than $T_{\mathrm{m}}$ and the nonlinear $I-V$ curve shows the decrease in the resistance. The same reasons determine the behavior of $I-V$ curve measured at $\tau_{\mathrm{i}}=10 \mathrm{~ns}$. It has to be noted that heating at high voltage regime is enough to reach temperatures at which $R-T$ dependence tends to saturation [4].

The investigation of wave forms of the current through the sample has demonstrated that switching process exhibits three phases (see Fig. 1b). The first phase (delay time) appearing immediately after the pulse rise time is a period during which relatively slow (few ns) increase in current takes place, the second phase (switching) is associated with abrupt (less than ns) jump, about ten times, in current and the last third phase (few ns) is the on-state formation period which is necessary to create time-independent low resistive state. The on-state could be maintained only if voltage across the film is higher than a certain value $V_{\text {on }}$. The increase in the overvoltage $\left(V-V_{\mathrm{th}}\right)$ decreases both delay and switching time.

The investigations show that after on-state formation the current in the interelectrode area is distributed non-uniformly. This can be seen form SEM picture 

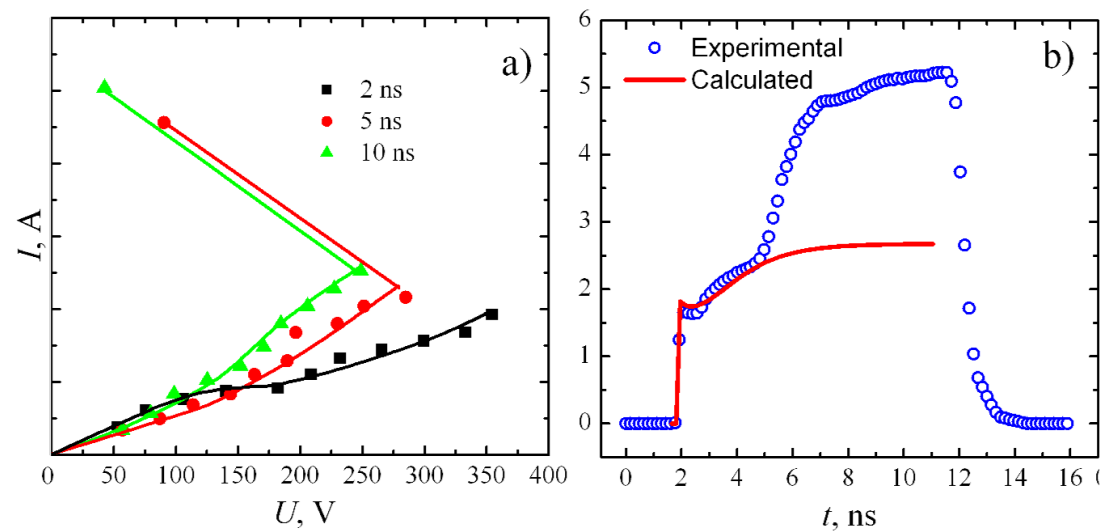

Fig. 1. (a) Current-voltage characteristics of $\mathrm{La}_{0.83} \mathrm{Sr}_{0.17} \mathrm{MnO}_{3}$ film measured at different time instants. $T=295 \mathrm{~K}$. (b) Switching dynamics at applied pulsed voltage across the sample with amplitude $V=450 \mathrm{~V}$.

demonstrating damage regions at the current electrodes (see Fig. 2a). The damage was concentrated in the narrow parts of the electrodes and manifested as melting of electrodes material in the interconnection region between $\mathrm{Ag}$ electrode and manganite film. The area of the damage region was always larger at the anode and narrowed at the cathode. Typical ratio between such "two-dimensional" channel width at the anode and cathode varied from 4 to 8 . Moreover, it has to be noted that high current in on-state damaged only electrode material, meanwhile the manganite film was not destroyed.
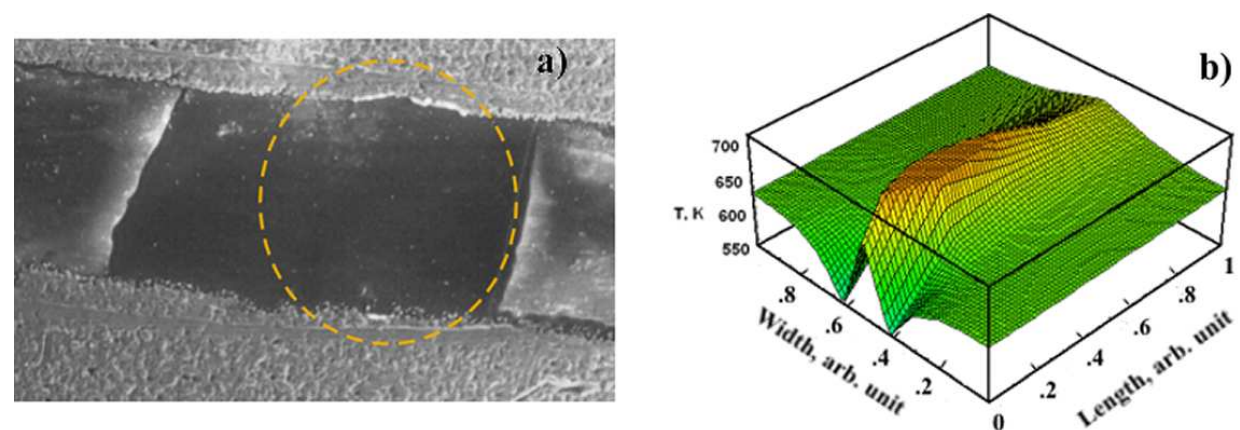

Fig. 2. (a) SEM picture demonstrating damage regions of $\mathrm{La}_{0.83} \mathrm{Sr}_{0.17} \mathrm{MnO}_{3}$ film at the current electrodes. (b) Simulated temperature distribution in the film along its length after $5 \mathrm{~ns}$.

The obtained phenomenon can be explained by the following model. During the first phase (delay time) high current induces fast heating of the manganite film and charge carriers are injected from cathode at a local region associated 
with imperfection of the Ag thin film electrode. These imperfections cover about 0.1 of the total interelectrode distance (see Fig. 2a). This led to appearance of highly localized low resistance region at the cathode. The further heating causes current crowding and increase in power dissipation at certain places of the interelectrode area. As a result, the high-current density channel is formed between electrodes. We simulated the manganite film as two-dimensional resistors ladder network with four series-parallel resistors of the same value in each element of the ladder and typical of manganite films resistance vs. temperature dependence, and obtained that peculiarities of current dynamics during delay time period can be well understood on the basis of electro-thermal model (see Fig. 1b, solid curve). The imperfection was introduced as a resistance located at the cathode and having three times smaller value comparing with the rest resistances of the network. This model also enabled to predict the asymmetrical shape of the current channel and showed that such shape is a result of non-homogeneous heating during the delay time (Fig. 2b). However, the thermal model was inconsistent to explain second phase, i.e. fast switching period. For this reason it has to be associated with electronic processes such as avalanche of the charge carriers in solid state material which was necessary to reach critical power in the interelectrode space. The calculations of the distribution of power dissipation in this area demonstrated that at the end of delay time the highest power is concentrated close to the anode. The asymmetrical damaging of the current electrodes could be an additional argument that electronic switching starts at the anode and then propagates to the cathode. However, the detailed mechanism of electronic switching has to be an object of further investigations.

\section{Conclusions}

It was concluded that the fast switching appearing in thin manganite film as a result of nanosecond duration strong electric field pulse action is of electronic nature. Meanwhile, the thermal processes occurring in high-current regime are responsible for the origin of the delay time and asymmetrical shape of current channel in on-state.

\section{Acknowledgments}

The work was supported by the Lithuanian Science and Studies Foundation contract No. B-21/2007.

\section{References}

[1] J. Gao, F.X. Hu, Appl. Phys. Lett. 86, 092504 (2005).

[2] N. Žurauskienè, Thin Solid Films 515, 576 (2006).

[3] A. Abrutis, V. Plausinaitiene, V. Kubilius, A. Teiserskis, Z. Saltyte, R. Butkute, J.P. Senateur, Thin Solid Films 413, 32 (2002).

[4] S. Balevičius, N. Žurauskienè, V. Stankevič, P. Cimmperman, S. Keršulis, A. Česnys, S. Tolvaišienè, L.L. Altgilbers, Appl. Phys. Lett. 90, 212503/1 (2007). 